\title{
SPONTANEOUS SUBARACHNOID HAEMORRHAGE IN CHILDREN
}

\section{A CLINICAL STUDY OF FIVE CASES}

BY

\author{
H. G. MILLER, M.B.
}

House Physician to the Royal Victoria Infirmary, Newcastle-upon-Tyne

The modern literature of subarachnoid haemorrhage may be said to begin with a classical paper by C. P. Symonds (1924), in which he describes the syndrome of haemorrhage into the subarachnoid space occurring spontaneously and apart from any injury or recognizable disease, and frequently traceable to a ruptured congenital aneurysm of the circle of Willis or one of its branches. Here is found correlated for the first time the subarachnoid haemorrhage of earlier writers (Gintrac, 1869 ; Hayem, 1872 ; Froin, 1904), with the detailed anatomical observations of Fearnsides (1916) on intracranial aneurysm. Since then several authors have amplified Symonds's work. Nattrass (1933) describes the various clinical pictures produced by bleeding from aneurysms of this nature ; and Taylor and Whitfield (1936) analyse eighty-one cases occurring in Birmingham, and make valuable observations on incidence, mortality, symptomatology and pathology.

References to the occurrence of spontaneous subarachnoid haemorrhage in children are few. In the general literature of the condition Nattrass (1933) comments on the apparent rarity of the syndrome in childhood, and one case in a child of ten is included in Taylor and Whitfield's series. Apart from these there are some isolated case reports of the condition occurring secondarily to other pathology. Thus Gintrac (1869) reports a case in a child of eighteen months secondary to superior longitudinal sinus thrombosis; between this time and the present day the French in particular have developed an extensive literature on the subject, of which an article by Traissac (1937) may be taken as representative. In a long paper on the subject he describes subarachnoid haemorrhage occurring in association with practically every disease that afflicts the child-infective fevers, blood diseases, tuberculosis, rheumatism and 'méningite lymphocytaire curable'; and attributes those cases which he recognizes to occur in children otherwise healthy to a haemorrhagic meningitis. This hypothesis is put forward in view of the absence of arteriosclerosis, atheroma and syphilis as causative factors in those children, and in apparent ignorance of the English work on intracranial aneurysm. He also propounds the theory that prognosis in these cases depends on the virulence of the activating and unrecognized infective agent.

The first reference to the condition occurring in children in the absence of other disease appears to be Bouquet's description (1908) of an apparently 
spontaneous attack, with recovery, in a child of fourteen years, following exposure to the sun. More recently Ramsay (1931) describes a case in a child of ten with premonitory symptoms, signs of meningeal irritation, bloody cerebrospinal fluid, and recovery ; and Barber (1936) describes a fatal case in an infant of five months with a spontaneous onset and a picture comparable to that of asphyxia pallida. Post-mortem examination in this case revealed a ruptured dissecting aneurysm of the circle of Willis, and in view of the history of a difficult forceps delivery the etiology of the aneurysm remains in doubt. Barber suggests that while spontaneous subarachnoid haemorrhages in children due to blood dyscrasia, sunstroke, or vasomotor disturbances have a good prognosis, those cases due to aneurysm or congenital deficiency of a vessel-wall are usually fatal : a conclusion which does not accord with what is known of the disease in adults.

Other case reports are those of Dyson (1932) - a spontaneous fatal case in a child of eleven ; and of Murray (1934) - a spontaneous case with recovery and residual symptoms in a boy of the same age.

\section{Case records}

The following five cases of subarachnoid haemorrhage, occurring in children apparently otherwise healthy, may therefore be of interest. Three of these cases have been seen by the author in Newcastle during the last few months, and particulars of the other two were obtained from the records of the Royal Victoria Infirmary.

Case 1. Thomas M., aged ten years, while playing football in March, 1935, was seized with sudden intense occipital headache and a paroxysm of copious vomiting. He walked home and on arrival there became unconscious, remaining so for two hours. Eighteen hours later, on admission to the Royal Victoria Infirmary, he was conscious but complained of intense pain in the back of the head and neck. He had neck stiffness and a positive Kernig's sign. The right pupil was larger than the left although both reacted to light; his optic fundi were normal, his deep reflexes brisk, and the plantar responses flexor ; he had a pulse rate of 120 , temperature $99.5^{\circ}$ F., B.P. $125 / 95$, and no albumin or sugar in the urine. Four days later the symptoms showed no improvement and lumbar puncture yielded six c.c. of uniformly blood-stained fluid under increased pressure, with some abatement of the severity of the headache. Sixteen days from the onset the temperature still remained between $99.5^{\circ}$ and $100.5^{\circ} \mathrm{F}$., although the symptoms had almost disappeared, neck stiffness was slight, and Kernig's sign absent. Seven days later the temperature fell to normal, neck rigidity had disappeared, and there were no neurological symptoms or signs. The blood pressure now read 110/85.

Seen in May, 1938, now aged thirteen years, this boy was perfectly well except for a discharging ear of some weeks' standing. He had never had a headache since leaving hospital, and examination was completely negative.

Here is the picture of a sudden, unheralded haemorrhage with a short period of unconsciousness and complete recovery, without residual symptoms, maintained for upwards of three years.

Case 2. Doreen B., aged eleven years, was the third child of a working miner, previously in perfect health and with no personal or family history of headache, migrainous attack, collapse or sudden death. Three weeks before admission to hospital she had complained of severe occipital headache which passed off completely in a few days. Ten days later she ran into the house from an errand, vomited her tea and fell to the ground, conscious, but with 
temporary ' loss of use of her legs,' which cleared up in ten minutes. She stayed in bed for three days and on the fourth day visited her doctor, who found nothing apparently wrong. During the next week she was rather listless, but ate well, went to school and did not complain of any headache.

At 2 p.m. on the day of admission, three weeks from the onset of the symptoms, she vomited her dinner, went out to play for an hour, came back into the house and vomited once more. She went to the lavatory and three minutes later was found insensible there, with some twitching of the left arm and rigid legs. She was admitted at 10 p.m. ; since her collapse eight hours previously she had been comatose and had uttered no cry.

On examination the patient was comatose, with limbs slightly flexed and neck extended. She was flushed and the pulse slow (60), full and bounding. The temperature was normal. Running down the midline of the forehead there was a subcutaneous capillary naevus $1 \frac{1}{2}$ inches long by $\frac{1}{2}$ inch broad.

Neck stiffness and Kernig's sign were both strongly positive, and elicitation of either caused the patient to open her eyes : the pupils were equal and responded to light, and examination of the optic fundi revealed early bilateral papilloedema. The triceps, knee and ankle-jerks were all brisk, the abdominal reflexes absent, and the plantar reflexes both showed yawning extensor responses.

There were no signs in any other system : the urine contained neither albumin nor sugar, and the blood pressure was 110/70.

On admission eight c.c. of uniformly blood-stained cerebrospinal fluid were obtained by lumbar puncture and the pulse rate immediately rose from 60 to 76 per minute. Left to settle, the fluid twelve hours later showed a dark sediment of red cells with coagulum-free straw-coloured supernatant fluid.

Seventy-two hours later the patient was still comatose but could swallow. The pulse rate had dropped to 66 , but rose to 80 on repetition of lumbar puncture. Fifteen c.c. of fluid were removed, similar in appearance to that previously obtained, but showing a brighter yellow supernatant fluid.

For six days after admission the patient's temperature was $100^{\circ}-100 \cdot 5^{\circ} \mathrm{F}$, and when the temperature fell she had come out of coma, but was apathetic and complaining of severe headache. She was able to feed herself; neck rigidity was still present, but the plantar reflexes were now equivocal. A Wassermann reaction taken was negative. After another three days the headache disappeared and with it the neck stiffness. There were now no neurological signs, with the exception of resolving choked discs, the plantar reflexes being clearly flexor, and the parents said the child was quieter than usual. During the next month the child gradually returned to complete normality, though the relatives said that her memory seemed impaired. She never at any time complained of further headache and was well two months after the attack (June 16, 1938) when she was discharged.

This history suggests a sudden big haemorrhage, with coma and recovery, following three weeks' premonitory symptoms, with probably at least one haemorrhage of lesser magnitude during that period. The facial haemangioma suggests the possibility of similar intracranial pathology as a source of the bleeding.

Case 3. Thomas N., aged three years, was a fatal case. The history was obtained from the mother's story at the inquest :

' On Tuesday, May 15, 1934, about 2.30 p.m. I heard him crying in the back lane. I went out and saw him holding his head. I asked him what was the matter and he said, "The baa-baa hit me." There were no signs whatever of injury and he soon stopped crying and appeared none the worse. The following day he was playing about and took his food quite well. About 4 a.m. on Thursday, May 17, he woke up crying. I coaxed him to sleep. At 6 a.m. he 
awoke crying again and I noticed his neck looked stiff. I thought it was the way he had been lying. He went to sleep again, but when I lifted him out of bed at $8 \mathrm{a} . \mathrm{m}$. he could not stand and his limbs were stiff. He very soon became unconscious, and was sent into the Infirmary that morning.'

On admission the child was unconscious with limbs all rigid and slightly flexed. His head was extended and stiff. He had one generalized clonic convulsion and died within an hour. Lumbar puncture was performed postmortem, and yielded almost pure blood. Unfortunately there was no autopsy, but in the presence of so definite a history there can be little doubt that this was a rapidly fatal case of spontaneous subarachnoid haemorrhage heralded by thirty-six hours' premonitory symptoms.

Case 4. George H., aged two years, previously perfectly healthy, was admitted to the Newcastle General Hospital on March 23, 1938, with the history of a pyrexial illness of twelve days duration. This began quite suddenly one evening with a bout of screaming, hot flushing, rapid respiration, and above all a marked resentment of being handled or touched in any way. The past and family histories were completely negative.

On examination, the child, flushed and resentful of interference, was lying on his side with his legs slightly flexed. There was some neck stiffness and a positive Kernig's sign, with no evidence of disease in any other system. The temperature was $101 \cdot 2^{\circ} \mathrm{F}$., respirations 28 , pulse rate 120 , examination of the urine revealed no abnormal constituents.

The next day neck rigidity was much more marked and by lumbar puncture under general anaesthesia, five c.c. of apparently pure blood were withdrawn. This settled into the characteristic two layers of corpuscles and supernatant coagulum-free yellow fluid. Five days later the puncture was repeated with a similar result : and a Wassermann reaction (blood) proved negative.

The child lay for fourteen days completely apathetic, being fed with difficulty ; and at the end of this time return to normality was accompanied by gradual loss of neck stiffness. After twenty-one days the continuous temperature of $100^{\circ}-102^{\circ} \mathrm{F}$., which had lasted in all for practically five weeks, fell to normal, and about the same time the child sat up. A week later the child walked and made a perfect recovery, being discharged quite well eight weeks after admission.

This case of prolonged illness without coma lasting six weeks would appear to suggest a gradual leak of blood into the subarachnoid space over at any rate some days, a syndrome not unfamiliar amongst the cases occurring in adults. Taylor and Whitfield (1936) report eleven of this type amongst their eighty-one cases, and it seems that where the headache is unaccompanied by loss of consciousness the prognosis is uniformly good.

Case 5. Elsie M., aged twelve, was admitted on March 12, 1936. Sixteen hours previously she was playing in the street and suddenly ran into the house holding her head, vomiting, and screaming. Sudden complete blindness followed, and in five minutes she collapsed unconscious into a chair.

At the age of two she was concussed after a fall on the head. She was unconscious for twelve hours and a left internal strabismus followed. She recovered completely in a few days. At the age of seven she had a severe attack of occipital headache with twitching over the right side. For the last six months she had been subject to epileptic fits, Jacksonian in type, starting with twitching of the face, followed by convulsive movements of the right arm and then the right leg. There was never any loss of consciousness. These attacks occurred about once a fortnight and there were usually three or four such fits : they responded to prominal.

On admission the patient was unconscious, the breathing stertorous, and there was general muscular rigidity. It was not recorded whether neck stiffness or Kernig's sign was present. The plantar responses were both extensor : 
there was a convergent squint, contracted pupils, and left papilloedema. The pulse rate was 104 and temperature $101^{\circ} \mathrm{F}$. Lumbar puncture revealed deeply blood-stained fluid under increased pressure, settling into the characteristic two layers and sterile on culture. A Wassermann reaction on venous blood proved negative. Death occurred forty-eight hours after the onset without recovery of consciousness. There was no autopsy.

The diagnosis here is by no means as clear as in the previous cases, particularly in view of the impossibility of a true evaluation of the history of trauma. Jacksonian attacks, excepting those of a transient nature during the onset of the catastrophe, appear to be rare in association with subarachnoid haemorrhage ; Taylor and Whitfield (1936) report one case preceded by nine months' left-sided attacks. However, in view of the history and the typical nature of the ultimate attack, the most probable diagnosis here seems to be subarachnoid haemorrhage from an intracranial aneurysm, following at least one earlier attack and preceded by local pressure signs.

\section{Commentary}

Although neither of the fatal cases came to autopsy, the diagnosis in every instance was confirmed by examination of the cerebrospinal fluid : and the clinical pictures in all five cases are so strikingly similar to those known to occur in adults that it is justifiable, at any rate in cases 1,3 and 4, where no complicating factors arise, to suspect a similar pathology. Intracranial aneurysm of the type that gives rise to subarachnoid haemorrhage in the healthy young adult has not frequently been recorded in children, but if, as structural evidence suggests, these aneurysms are truly congenital in nature, they must obviously be present (perhaps on occasion only as an initial medial weakness) at birth. The frequently reported association between intracranial aneurysm and coarctation of the aorta is further evidence in favour of a congenital origin, and Fearnsides reports one significant case of a definite aneurysm of the circle of Willis found at autopsy in a child of nineteen months dying of bronchopneumonia complicating gastritis.

In view, therefore, of the clinical findings detailed above, it is suggested that in three of these cases the haemorrhage was in all probability due to rupture of a congenital intracranial aneurysm, and that pathologically as well as clinically these cases are analogous with spontaneous subarachnoid haemorrhage occurring in adults.

Clinically, one or two points of interest arise.

Occupation at onset.- In those children with a definite history of a sudden onset of the illness, the occupation of the child at the probable onset of the haemorrhage was known : three (cases $1,3,4)$ were playing out of doors and one (case 2) ' ran in from an errand.' In this case the later haemorrhage with loss of consciousness occurred while the child was at stool. As demonstrated by Taylor and Whitfield (1936), effort appears to be a factor in provoking haemorrhage into the subarachnoid space.

Pyrexia.- The above authors also found pyrexia, usually between $99^{\circ}$ and $101^{\circ} \mathrm{F}$., in all their cases which survived sufficiently long; and in no case without clinical recurrence of the haemorrhage did this pyrexia last longer than seven days. Amongst the five cases in children reported here, one ran a 
temperature of $100^{-}-102^{2} \mathrm{~F}$. for five weeks (case 4), this being diagnosed as probably a slow leak; and in another case (case 1) in which there was no reason to suspect that a recurrence of haemorrhage took place after the first dramatic attack, the temperature persisted at $99 \cdot 5^{\circ}-101 \cdot 5^{2} \mathrm{~F}$. for sixteen days. Such variation in so small a number of cases suggests that in children the heatregulating mechanism is not so stereotyped in its reaction to the haemorrhage as in adults, a fact which in the absence of lumbar puncture would further complicate the differential diagnosis from some form of meningitis.

Premonitory symptoms. - Of the four cases with a well-defined onset and unconsciousness, three showed some premonitory symptoms-headache (cases 2, 3) and Jacksonian attacks (case 5).

Urine.-Neither glycosuria nor albuminuria was present in any of the three cases in which the urinary findings were recorded.

Prognosis.-From the point of view of prognosis nothing can be added to what has been written of the disease as it occurs in adults, except that residual symptoms were not observed in any of recovered cases (cases 1, 2, 4) during the time since their haemorrhages; and that, judging from case 1 , the ultimate prognosis may not be essentially unfavourable.

\section{Summary}

Five cases of spontaneous subarachnoid haemorrhage, two fatal, and all confirmed by lumbar puncture, are described in children between the ages of two and twelve years. They present various clinical pictures strictly comparable to those known to occur in this disease as it affects adults. In one case the presence of a facial haemangioma leads to the suspicion of some similar intracranial pathology as a source of the haemorrhage, and in three of the other four cases the presence of an aneurysm, probably congenital, is suspected. The rôle of effort in the provocation of the attack and the frequency of premonitory symptoms are illustrated, and the pyrexial phenomena associated with the haemorrhage are shown to be somewhat inconstant as compared with the findings in adults.

Thanks are due to members of the medical staffs of the Royal Victoria Infirmary and the General Hospital, Newcastle-upon-Tyne, for kindly allowing access to their cases and records and for permission to publish this material.

\section{REFERENCES}

Barber, W. W. (1936). Colorado Medicine, 33, 182.

Bouquet, (1908). Province Med., 1, 398.

Dyson, J. E. (1932). J. Iowa Med. Soc., 22, 223.

Fearnsides, C. G. (1916). Brain, 39, 224.

Froin, (1904). Thèse de Paris.

Gintrac, (1869-71). Maladies de l'appareil Nerveux, Paris, 1, 649.

Hayem, (1872). Thèse de Paris.

Murray, M. F. (1934). Clin. Miscell. ; Mary I. Basset Hosp., 1, 89.

Nattrass, F. J. (1933). Lancet, 2, 915.

Ramsay, M. L. (1931). Brit. med. J., 1, 1,070.

Symonds, C. P. (1924-5). Quart. J. Med., 18, 93.

Taylor, A. B., and Whitfield, A. G. W. (1936). Ibid., 5, 461.

Traissac, M. (1937). J. Med. de Bordeaux, 32, 1,142. 